\title{
Tracking Ultrafast Fluorescence Switch-on and Color-tuned Dynamics in Acceptor-donor- acceptor Chromophore
}

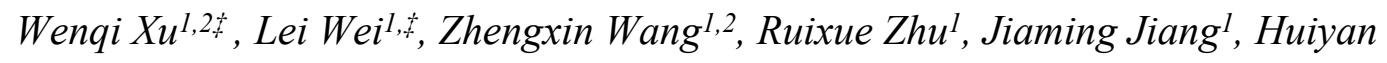 \\ Liu, ${ }^{1}$ Juan Du ${ }^{3}$, Tsu-Chien Weng, ${ }^{1}$ Yuebiao Zhang ${ }^{1,}{ }^{*}$, Yifan Huang ${ }^{1,{ }^{*}}$ and Weimin

$$
\operatorname{Liu}^{1,2, *}
$$

1. School of Physical Science and Technology, ShanghaiTech University, Shanghai 201210, People's Republic of China.

2. STU and SIOM Joint Laboratory for Superintense Lasers and the Applications, Shanghai 201210, People's Republic of China.

3. State Key Laboratory of High Field Laser Physics, Shanghai Institute of Optics and Fine Mechanics, Chinese Academy of Sciences, Shanghai 201800, China.

$\$$ These authors contributed equally to this work 
*Authors to whom correspondence should be addressed: liuwm@shanghaitech.edu.cn; huangyf@shanghaitech.edu.cn and zhangyb@shanghaitech.edu.cn

\section{Contents}

S1. Synthesis of 4,4'-(2,3,6,7-tetramethoxyanthracene-9,10-diyl)dibenzaldehyde

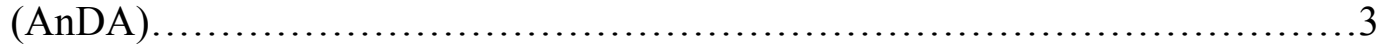

S2. Synthesis of 2,3,6,7-tetramethoxy-9,10-diphenylanthracene (TMDPAn)......3

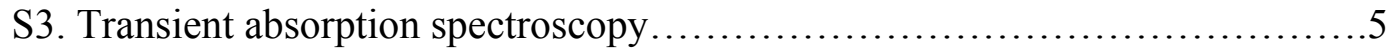

S4. Time-resolved up-conversion fluorescence spectroscopy..................5

S5. Femtosecond stimulated Raman spectroscopy (FSRS)....................6

S6. Calculations.....................................................6

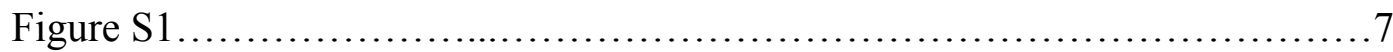

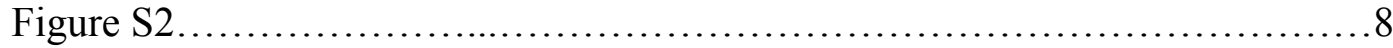

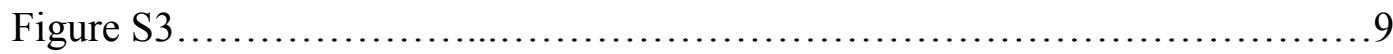

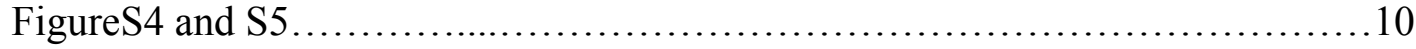

S7. Transient absorption spectra of AnDA crystalline film.....................11

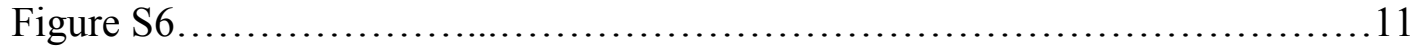

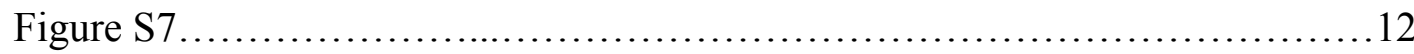

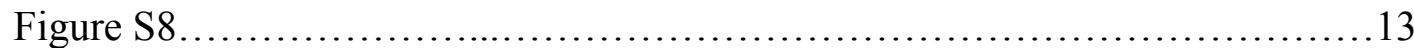

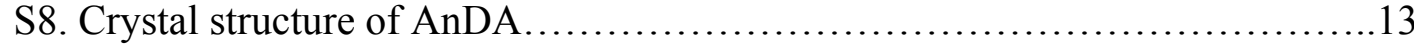

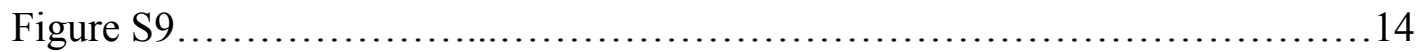

Figure S10 and S11.................................................... 15

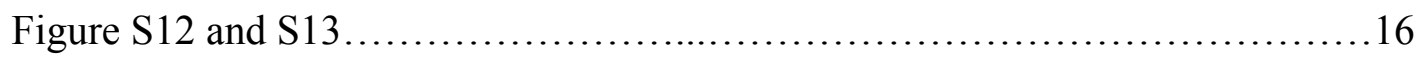

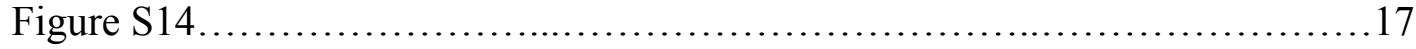

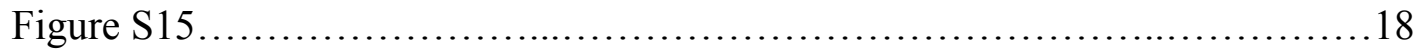

Figure S16 and S17 .................................................... 19

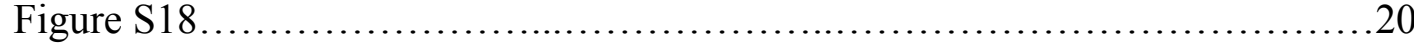




\section{S1. Synthesis of 4,4'-(2,3,6,7-tetramethoxyanthracene-9,10- diyl)dibenzaldehyde (AnDA):}

A solution of veratrole (40 mmol, 1 equiv, $5.36 \mathrm{~g}$.) and the terephthalaldehyde (40 mmol, 1equiv, 5.364 g.) in dichloromethane $(30 \mathrm{~mL})$ was added dropwise to $84 \%$ sulfuric acid $(40 \mathrm{~mL})$ while the temperature was kept between 0 and $5^{\circ} \mathrm{C}$. After the addition, the suspension was stirred for $2.0 \mathrm{~h}$ at room temperature. The reaction was quenched with water and neutralized by ammonia. After extraction with dichloromethane, the solvent was stripped off in vacuum. Then the crude product was purified by chromatographically with $\mathrm{CHCl}_{3}$ and ethyl acetate, affording $\mathrm{AnDA}$ in $20 \%$ yield; ${ }^{1} \mathrm{H}$ NMR (400 MHz, $\left.\mathrm{CDCl}_{3}\right) \delta 10.24(2 \mathrm{H}, \mathrm{s}, \mathrm{CHO}), 8.19(4 \mathrm{H}, \mathrm{d}, J=8.0 \mathrm{~Hz}, 2-$ 6-H phenyl), 7.71 (4H, d, $J=8.0 \mathrm{~Hz}, 3-5-\mathrm{H}$ phenyl), 6.73 (4H, s, 1,8-H anthracene), $3.74\left(12 \mathrm{H}, \mathrm{s}, \mathrm{OCH}_{3}\right) .{ }^{13} \mathrm{C} \mathrm{NMR}\left(100 \mathrm{MHz}, \mathrm{CDCl}_{3}, \mathrm{ppm}\right) \delta 192.152,149.302,146.696$, 135.667, 132.019, 131.915, 130.249, 125.359, 103.352, 55.591.; MS (EMI) m/z: 507.1785 .

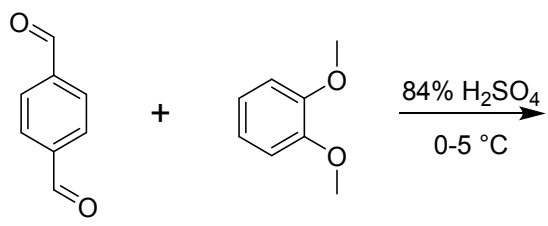

TPA<smiles>COc1cc2c(-c3ccc(C=O)cc3)c3cc(OC)c(OC)cc3c(-c3ccc(C=O)cc3)c2cc1OC</smiles>

AnDA

\section{S2. Synthesis of 2,3,6,7-tetramethoxy-9,10- diphenylanthracene (TMDPAn):}

A solution of veratrole $(2.2 \mathrm{~g}, 16 \mathrm{mmol})$ and the benzaldehyde $(2.55 \mathrm{~g}, 24 \mathrm{mmol})$ in dichloromethane $(1.5 \mathrm{ml})$ was added dropwise to $84 \%$ sulfuric acid $(3 \mathrm{ml})$ while the temperature was cooled at $0-5^{\circ} \mathrm{C}$. Further stirred for $15 \mathrm{~h}$ at room temperature and then the solvent was stripped off in vacuum. The crude solid was washed with mixture solution of alcohol and 
diethyl ether for several times, affording DPTMAn $1.55 \mathrm{~g}(3.4 \mathrm{mmol}) .{ }^{1} \mathrm{H}$ NMR (400 MHz, $\left.\mathrm{CDCl}_{3}\right) \delta 7.60(4 \mathrm{H}, \mathrm{t}, J=7.6 \mathrm{~Hz}), 7.52(2 \mathrm{H}, \mathrm{d}, J=7.5 \mathrm{~Hz}), 7.48(2 \mathrm{H}, \mathrm{d}, J=7.4 \mathrm{~Hz})$, $6.82(4 \mathrm{H}, \mathrm{s}),, 3.73\left(12 \mathrm{H}, \mathrm{s}, \mathrm{OCH}_{3}\right)$.

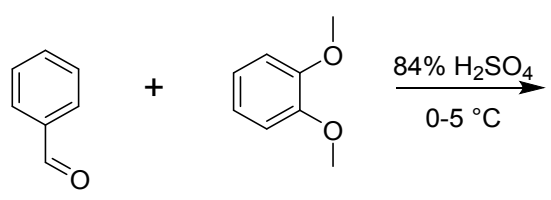

BA<smiles>COc1cc2c(-c3ccccc3)c3cc(OC)c(OC)cc3c(-c3ccccc3)c2cc1OC</smiles>

TMDPAn 


\section{S3. Transient absorption spectroscopy}

Transient absorption (TA) spectra in fs to ns region were collected using commercial transient absorption spectrometer (HELIOS, Ultrafast System). The 800 $\mathrm{nm}$ fundamental pulses were generated from a Ti: Sapphire laser system (Coherent, Astrella, $35 \mathrm{fs}, 7 \mathrm{~mJ} /$ pulse, $1 \mathrm{kHz}$ repetition rate.). A $375 \mathrm{~nm}$ pulse was used as the actinic pump for our TA experiment, which was generated by $800 \mathrm{~nm}$ fundamental pulses via an optical parametric amplifier (OPerA Solo, Coherent Inc.). The actinic pump power was then reduced via a neutral density filter after an optical chopper operating at $500 \mathrm{~Hz}$. A broadband supercontinuum white light with wavelength range from $\sim 450 \mathrm{~nm}$ to $\sim 800 \mathrm{~nm}$ serves as the probe pulse, which was obtained by focusing the fundamental beam into a Sapphire windows and the time window limit is $7 \mathrm{~ns}$.

EOS-TA spectra in ns- $\mu$ s region were collected using commercial transient absorption spectrometer (EOS, Ultrafast System). A $375 \mathrm{~nm}$ actinic pump pulse was generated by $800 \mathrm{~nm}$ fundamental pulses via an optical parametric amplifier (OPerA Solo, Coherent Inc.). A broadband supercontinuum white light with wavelength range from $\sim 320 \mathrm{~nm}$ to $\sim 900 \mathrm{~nm}$ serves as the probe pulse, which was obtained through a subns white light laser. The instrument response function (IRF) of this EOS system was determined to be $\sim 100 \mathrm{ps}$.

\section{S4. Time-resolved up-conversion fluorescence spectroscopy}

The femtosecond fluorescence up-conversion spectra experiments were performed based on a commercial femtosecond Ti/Sapphire regenerative amplifier laser system (Vitara, Astrella, Coherent Inc. $35 \mathrm{fs}, 7 \mathrm{~mJ} /$ pulse, $1 \mathrm{kHz}$ repetition rate.) and an automated data acquisition time-resolved fluorescence up-conversion spectrometer (Halcyone Fire, Ultrafast Systems). Part of the fundamental pulse $(2.0 \mathrm{~mJ} / \mathrm{pulse})$ were input to an optical parametric amplifier (TOPAS, Coherent Inc.) to obtain actinic pump puls at $375 \mathrm{~nm}$, then the actinic pump was focused on sample to generate fluorescence. A small portion of $800 \mathrm{~nm}$ fundamental pulse were used as gate pulse, fluorescence was collected using a pair of parabolic focus mirrors and then focused into a Beta-BaB2O4 
(BBO) crystal, which was used for sum frequency with the $800 \mathrm{~nm}$ gate pulse. Upconversion signals were collected using a monochromator and detected using a CCD camera. The time resolution was determined to be $250 \mathrm{fs}$.

\section{S5. Femtosecond stimulated Raman spectroscopy (FSRS)}

About $7 \mathrm{~mJ}$ of the fundamental laser pulses (Coherent, Astrella, $35 \mathrm{fs}, 7 \mathrm{~mJ} /$ pulse, $1 \mathrm{kHz}$ repetition rate.) was split into three beams to generate a tunable narrowband picosecond (ps) Raman pump, a broadband femtosecond (fs) Raman probe, and an fs actinic pump. The actinic pump centered at $375 \mathrm{~nm}$ with average power of $\sim 700$ $\mathrm{nJ} /$ pulse was generated from optical parametric amplifier (OPerA Solo, Coherent Inc.) for excited-state FSRS. About $15 \mu \mathrm{J} /$ pulse of the fundamental pulses is focused onto a Z-cut single-crystal sapphire plate to obtain a supercontinuum white light as Raman probe. For our tunable Raman pump, $\sim 3 \mathrm{~mJ}$ of fundamental pulses is directed through a second harmonic bandwidth compressor (SHBC, Coherent Inc.) produces a $400 \mathrm{~nm}$ pulse to pump the ps optical parametric amplifier system (TOPS400, Coherent Inc.). The instrument response time is measured by the cross correlation between the fs actinic pump and Raman probe pulses to be $\sim 150$ fs.

\section{S6. Calculations}

The geometries of AnDA and TMDPAn at ground state and $1^{\text {st }}$ excited state were optimized by using density functional theoretical methods. The Raman spectra calculation were carried out on the optimized geometry at ground state by using the functional of B3LYP. The fluorescence spectra of both molecules were calculated on the optimized $1^{\text {st }}$ excited states by using time-dependent density functional theoretical methods, where the functional of PBE1PBE were used. In the calculations of ground state and $1^{\text {st }}$ excited state, the basis set of $6-311+\mathrm{G}^{* *}$ was applied on the elements of $\mathrm{C}, \mathrm{O}$ and $\mathrm{H}$. The solvation effect was described by the polarizable continuum model (PCM). All calculations were performed by using Gaussian 09E ${ }^{1}$. 

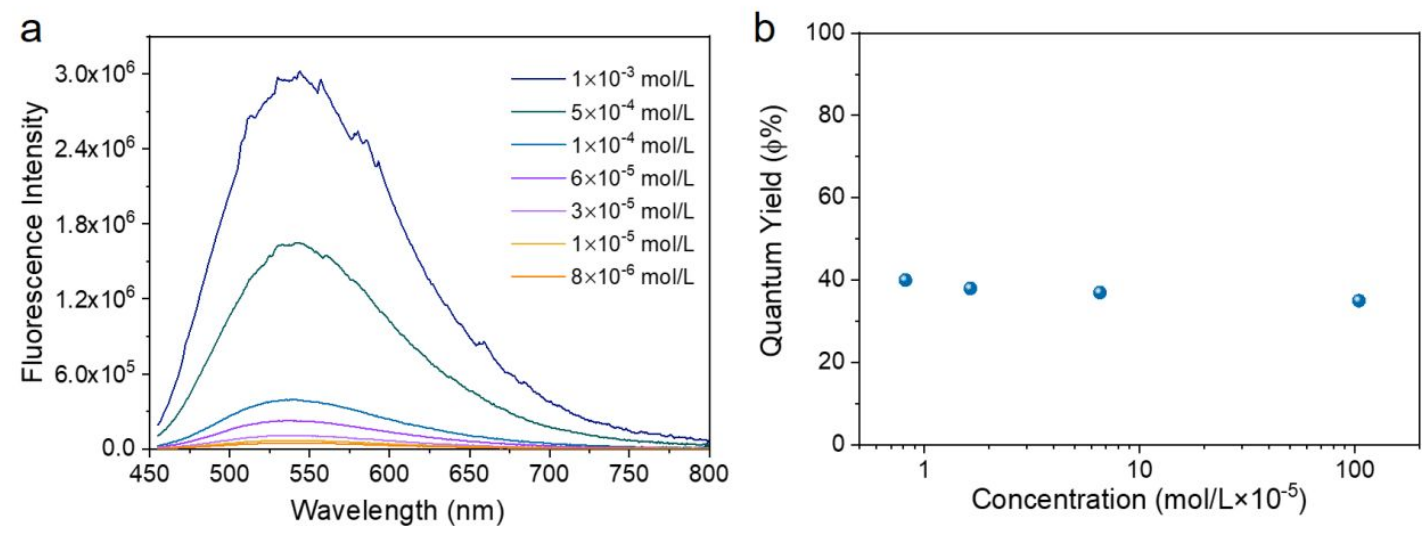

Figure S1. (a) concentration-dependent fluorescence spectra of AnDA and (b) concentration-dependent quantum yield of AnDA. 

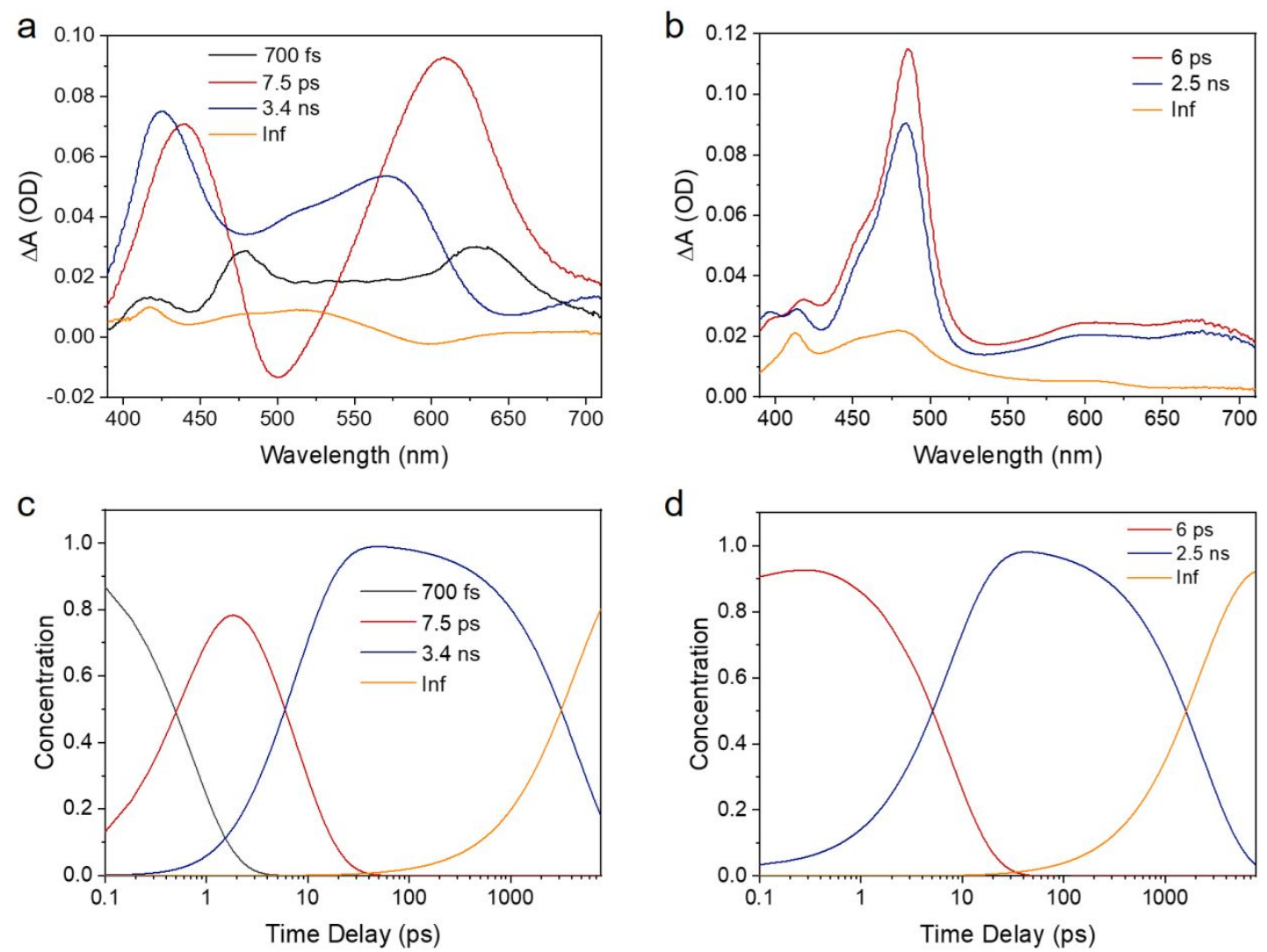

Figure S2. The extracted EADS components evolution from TA for (a) AnDA and (b) TMDPAn; Concentrations of transient species as a function of time for (c) AnDA and (d) TMDPAn. 

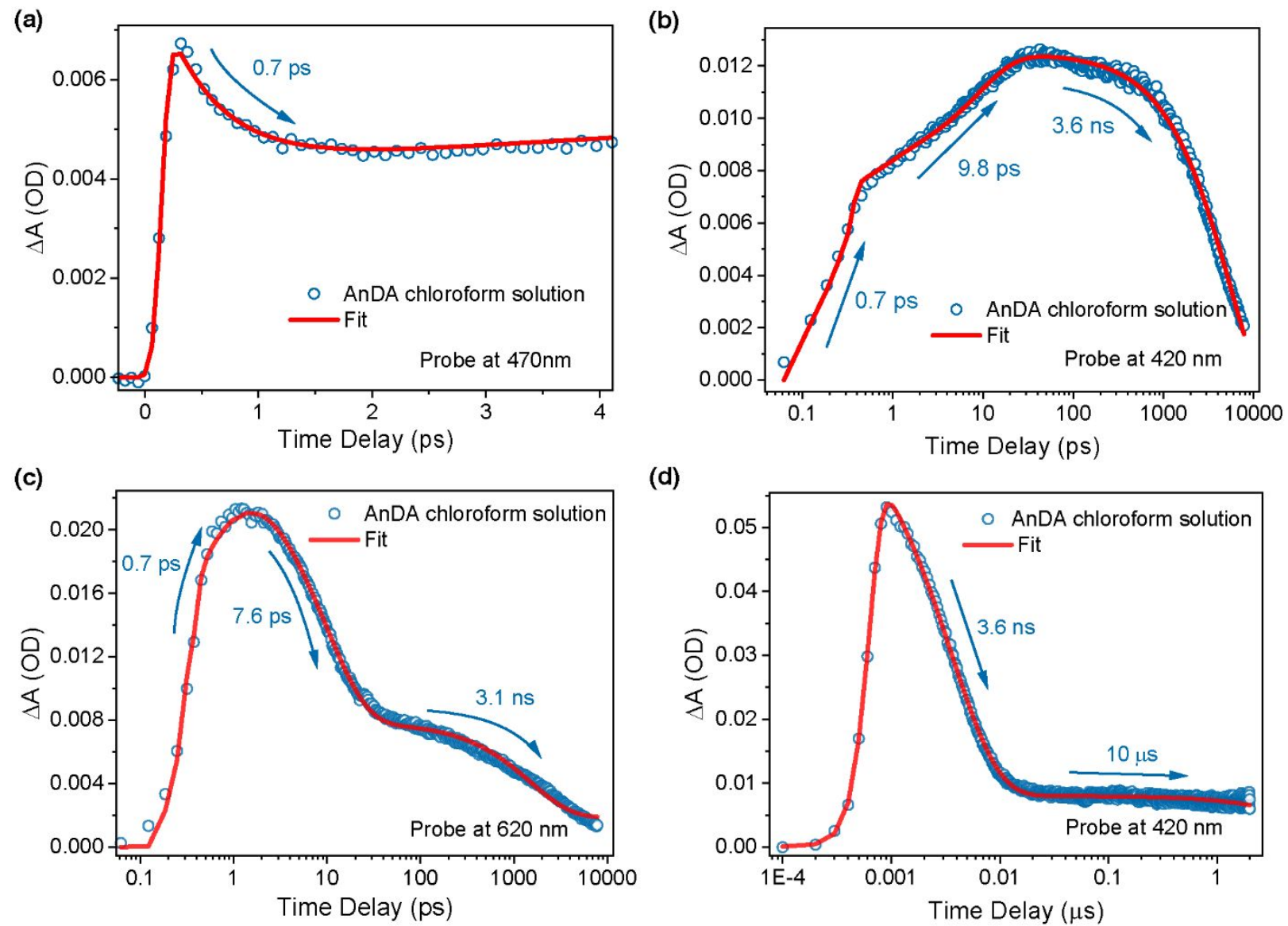

Figure S3. AnDA TA kinetic traces at (a) $470 \mathrm{~nm}$, (b) $420 \mathrm{~nm}$ and (c) $620 \mathrm{~nm}$; and (d) AnDA EOS-TA kinetic trace at $420 \mathrm{~nm}$. 


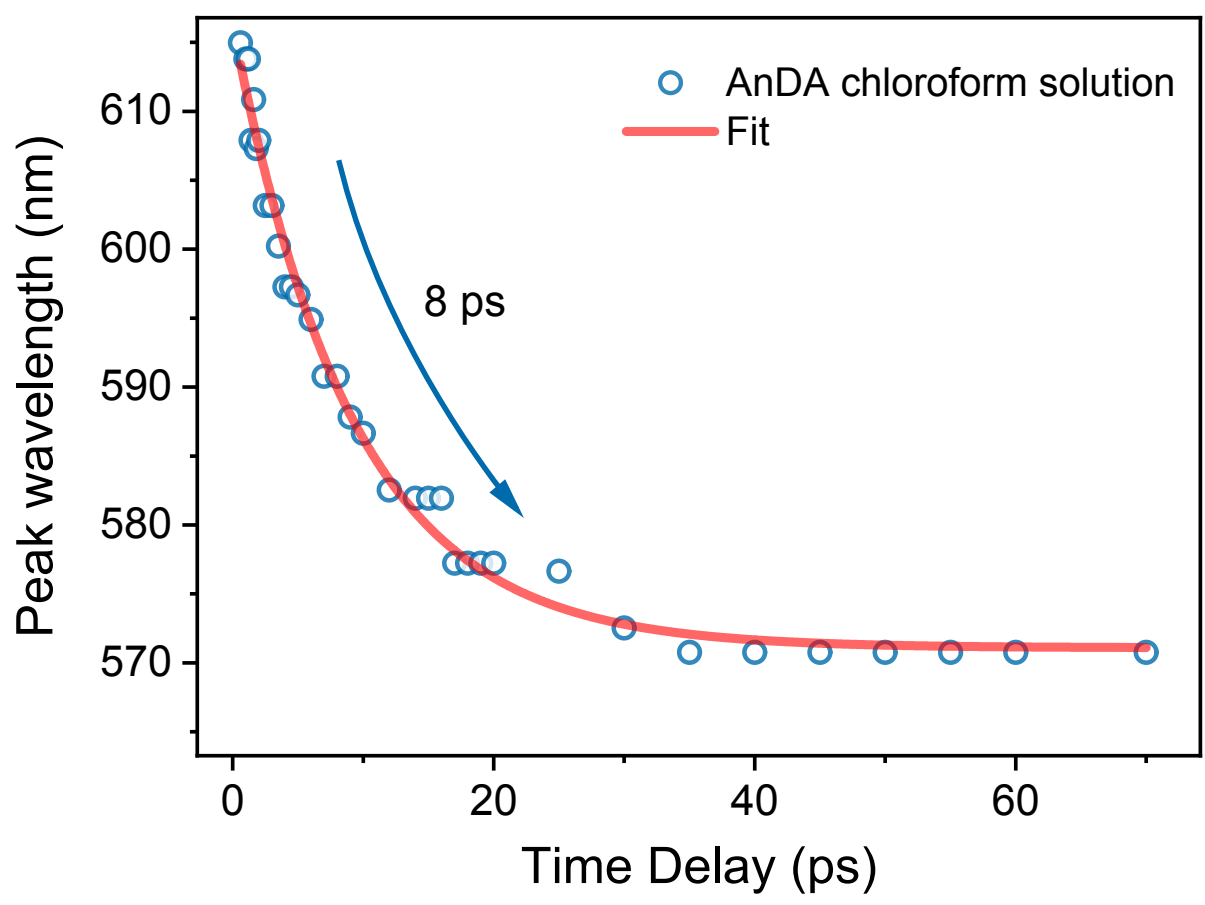

Figure S4. The frequency shift dynamics of ESA band at $620 \mathrm{~nm}$ for AnDA.

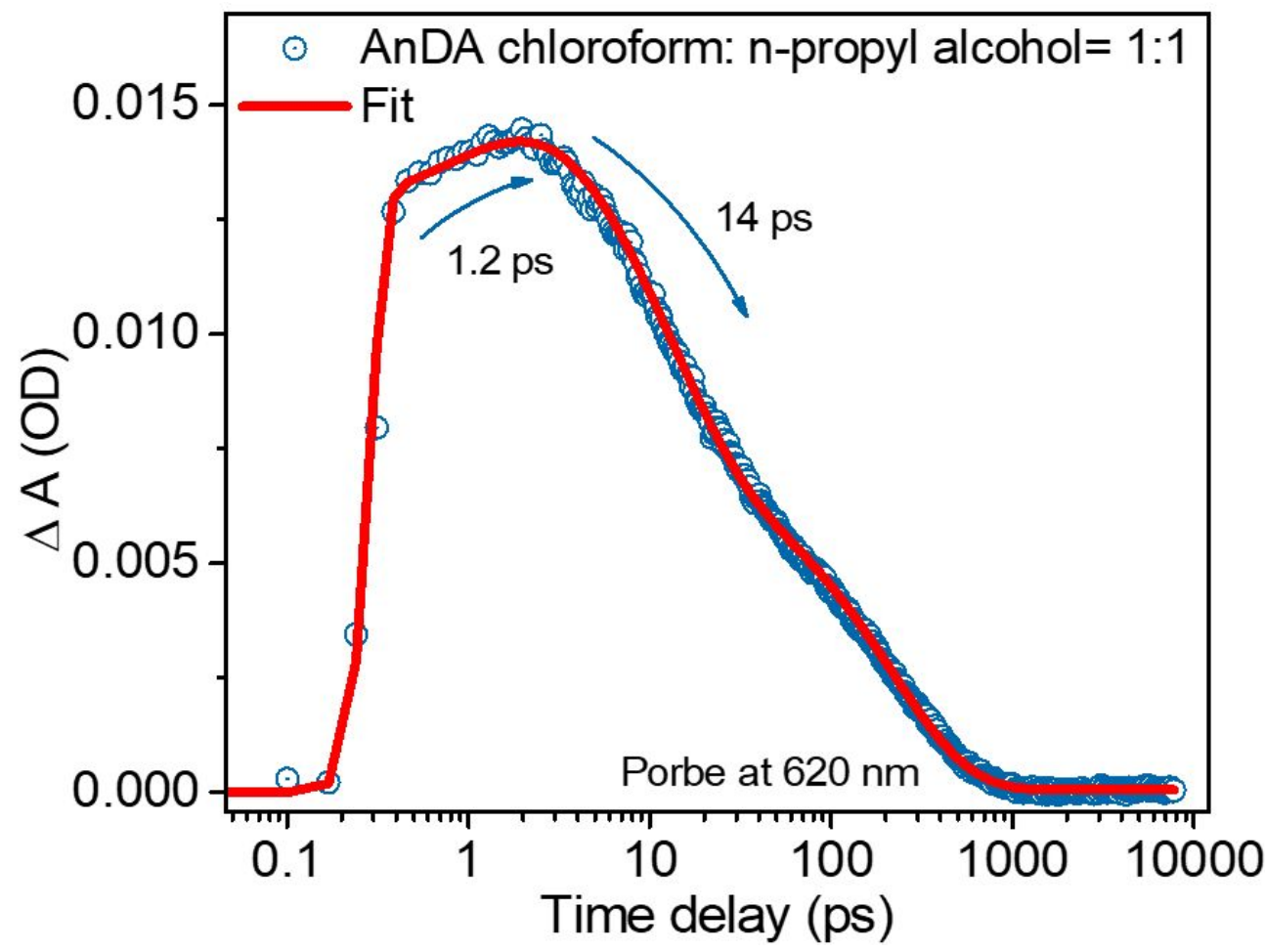

Figure S5. TA kinetic traces of AnDA in chloroform +n-propyl alcohol (1:1) at 620 nm. 


\section{S7. Transient absorption spectra of AnDA crystalline film}

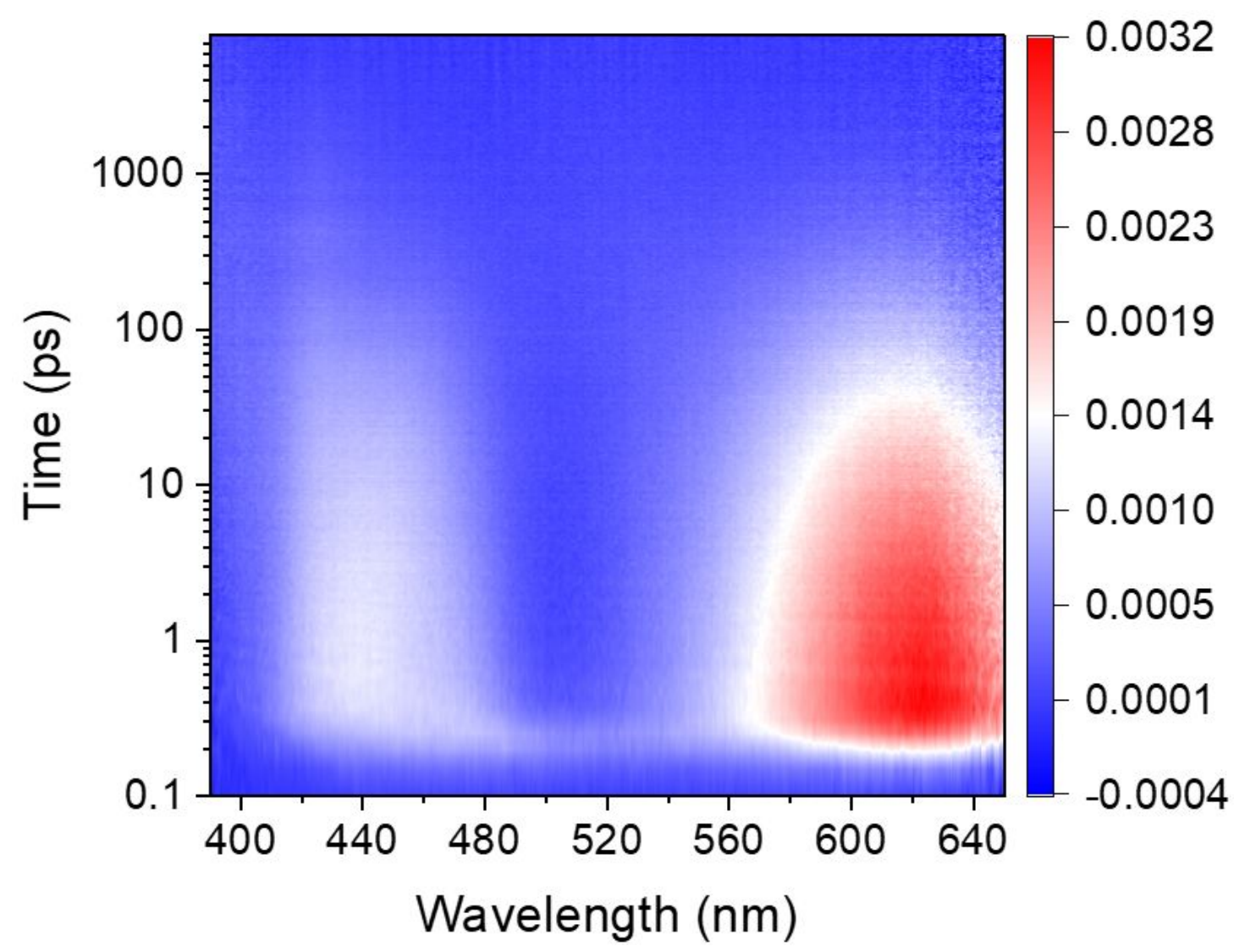

Figure S6. 2D Contour plot of TA spectroscopy of AnDA crystalline film following $375 \mathrm{~nm}$ excitation.

Ultrafast two-dimension TA spectra of the AnDA crystalline film under the excitation of $375 \mathrm{~nm}$ is shown in Fig S4. TA spectrum exhibits dominant two ESA bands center at the $450 \mathrm{~nm}$ and $620 \mathrm{~nm}$ range. The excited state decay dynamics of the ESA band at $620 \mathrm{~nm}$ is attributed to the IC from the LE to ICT state upon photoexcitation. Fig S5 shows a comparison of the TA kinetic trace between AnDA in chloroform and AnDA crystalline film at $620 \mathrm{~nm}$ under the excitation of $375 \mathrm{~nm}$. For AnDA in chloroform, the excited state dynamics exhibit an ultrafast rise dynamics with a time constant of $0.78 \mathrm{ps}$ followed by three exponential decay processes. The ultrafast rise $(0.78 \mathrm{ps})$ at $620 \mathrm{~nm}$ indicates the fluorescence switch-on dynamic via the transition 
of LE state to the ICT state, which is associated with the deviation of the dihedral angle between TMAn and BA moieties from the orthogonal geometry. Different with AnDA in chloroform, however, this ultrafast rise component was not observed from AnDA crystalline film at a probe wavelength of $620 \mathrm{~nm}$, as shown in Figures S5. Combined with the crystal structure of AnDA crystalline film shown in Fig S6, the excited state dynamics of AnDA crystalline film indicates direct formation of ICT state upon vertical photo-excitation due to non-orthogonal configuration.

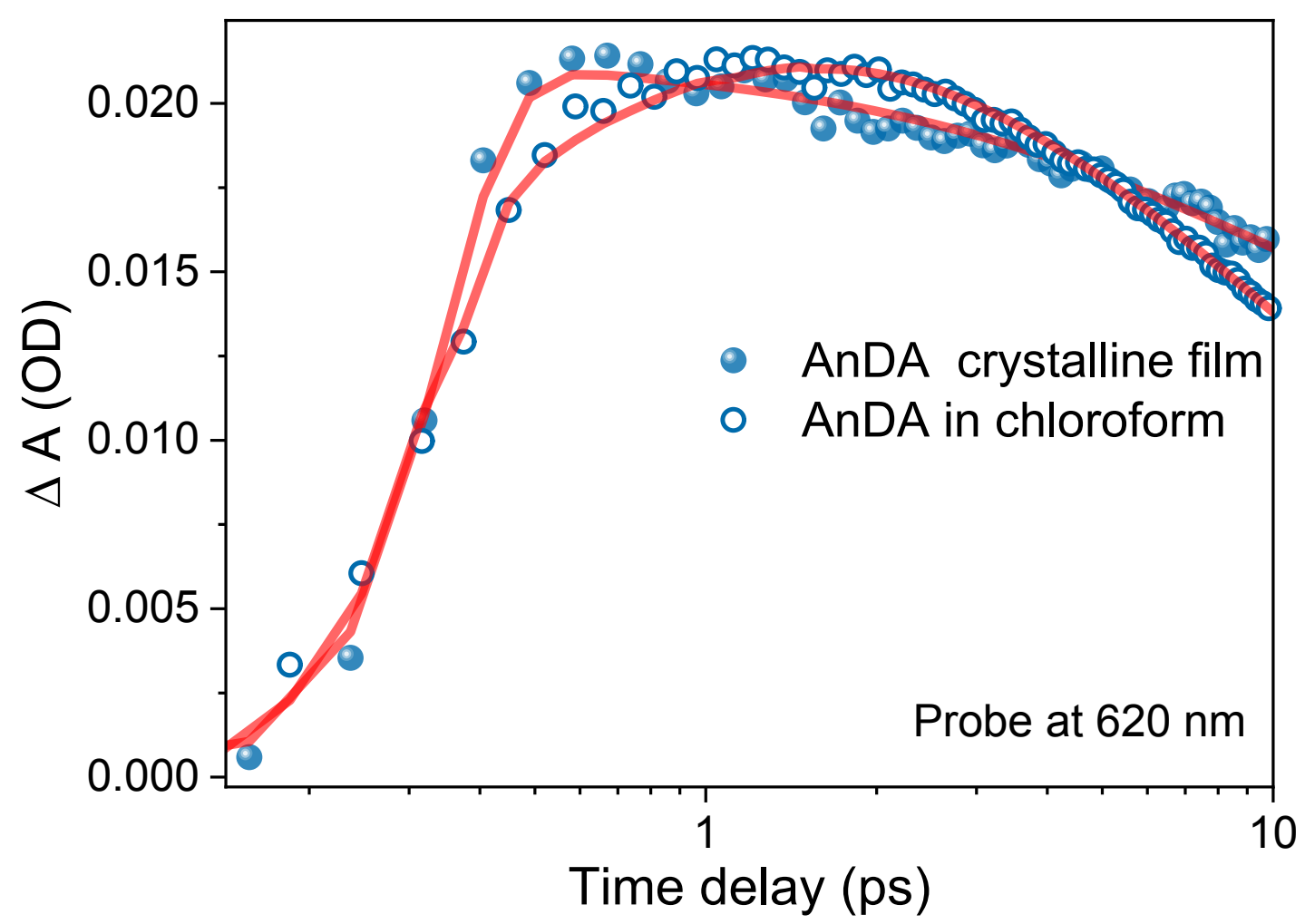

Figure S7. TA kinetic traces of AnDA in chloroform (black open circle) and AnDA crystalline film (black solid circle) at $620 \mathrm{~nm}$. 


\section{S8. Crystal structure of AnDA}

Yellow-colored single crystals were obtained by slow evaporation of a solution of AnDA in chloroform. The crystal structure of AnDA was revealed by single-crystal Xray analysis, the dihedral angle of phenyl and anthracene core is $69.1^{\circ}$ and $69.9^{\circ}$ (Figure S6). The distance between the oxygen of methoxy moist and hydrogen of adjacent phenyl moist are $2.40,2.69,2.45,2.46 \AA$, respectively, indicating that exist hydrogen bond interaction (Figure S7).

Preparation of AnDA film. Glass sheets with a size of $2 \mathrm{~cm} \times 2 \mathrm{~cm}$ were washed with water, ethanol, and $i$-propanol three times. A solution of dissolved $20 \mathrm{mg}$ AnDA in 1 $\mathrm{mL}$ chloroform was prepared. The spin-coating process was used for film preparation at a rate of $400 \mathrm{rad} / \mathrm{min}$

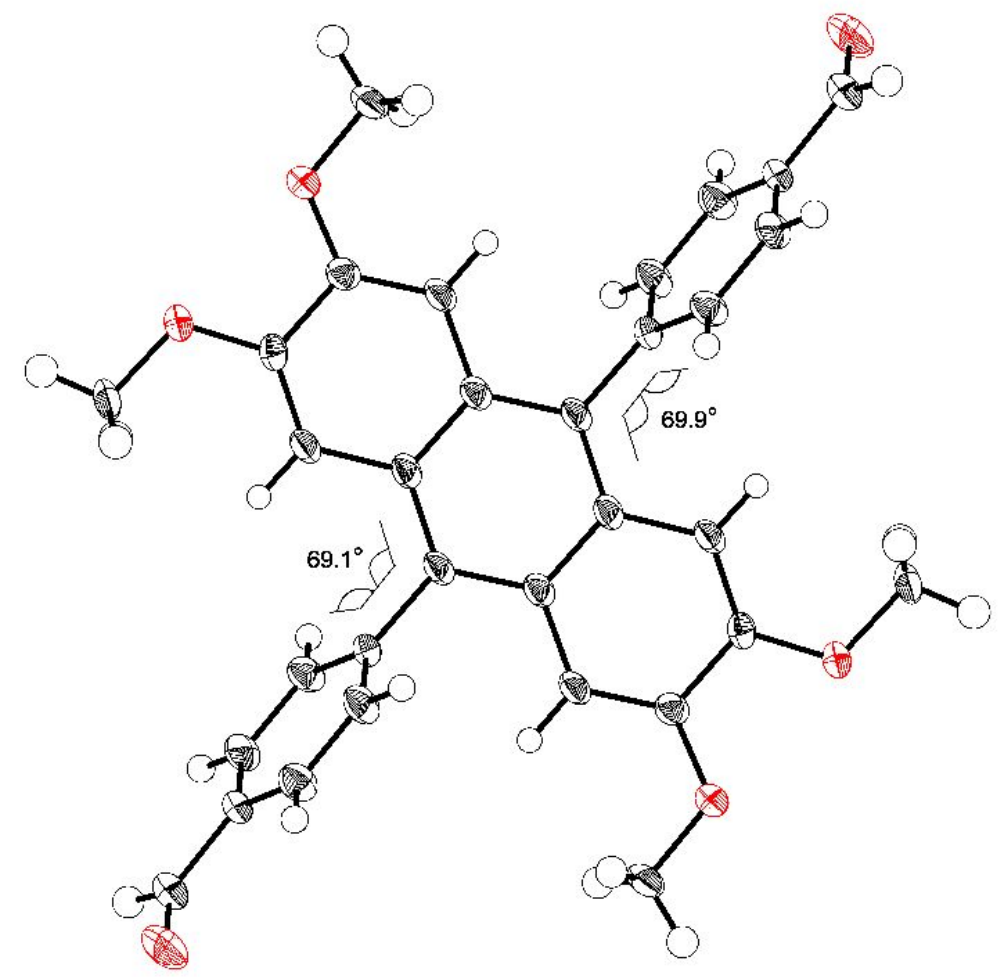

Figure S8. ORTEP drawing of the crystal structure of AnDA. Thermal ellipsoids are displayed with $50 \%$ probability. 


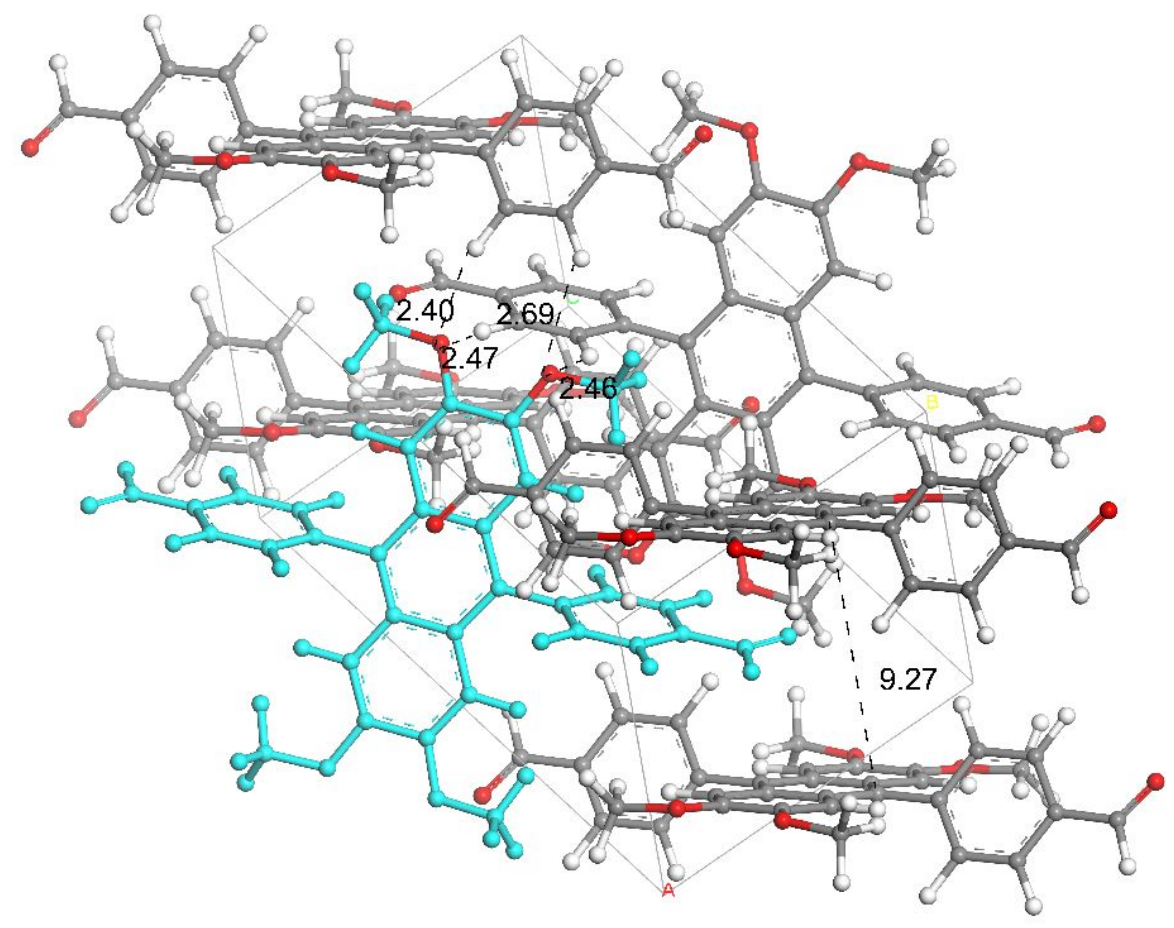

Figure S9. Single-crystal analyses and packing patterns of AnDA. The distance between two adjacent anthracene is $9.27 \AA$. 


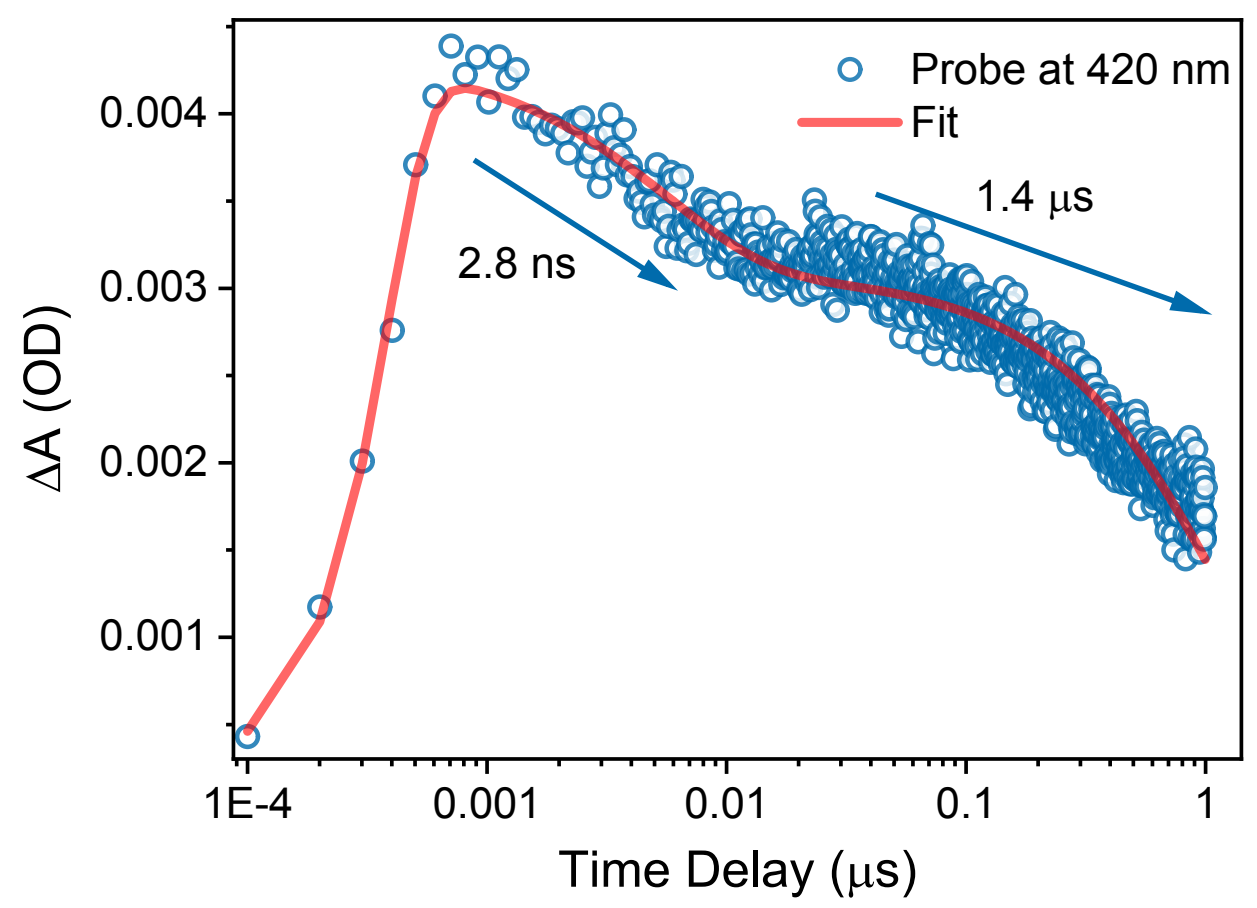

Figure S10. EOS-TA kinetic trace of TMDPAn at $420 \mathrm{~nm}$.

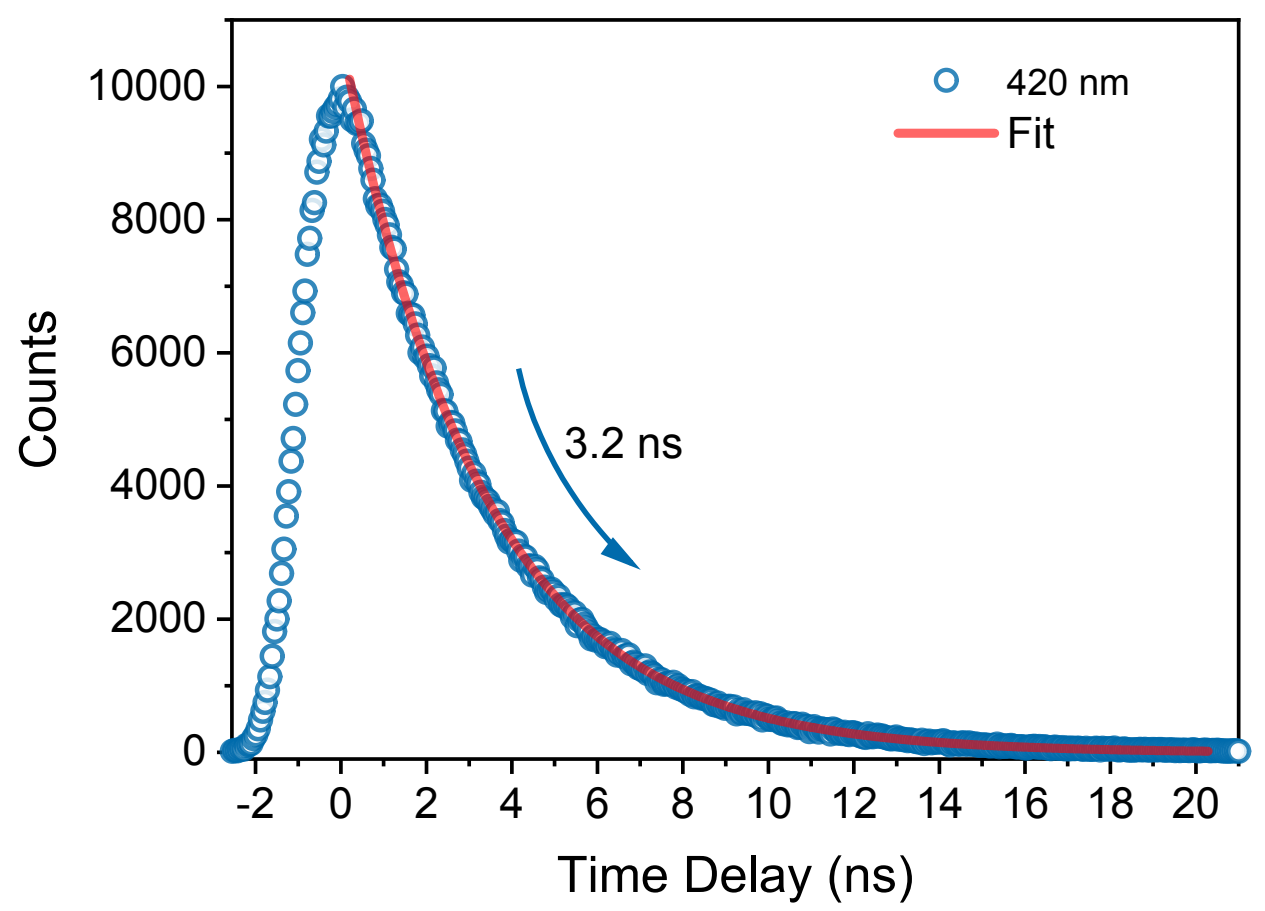

Figure S11. Time-resolved fluorescence decays of TMDPAn in chloroform. 


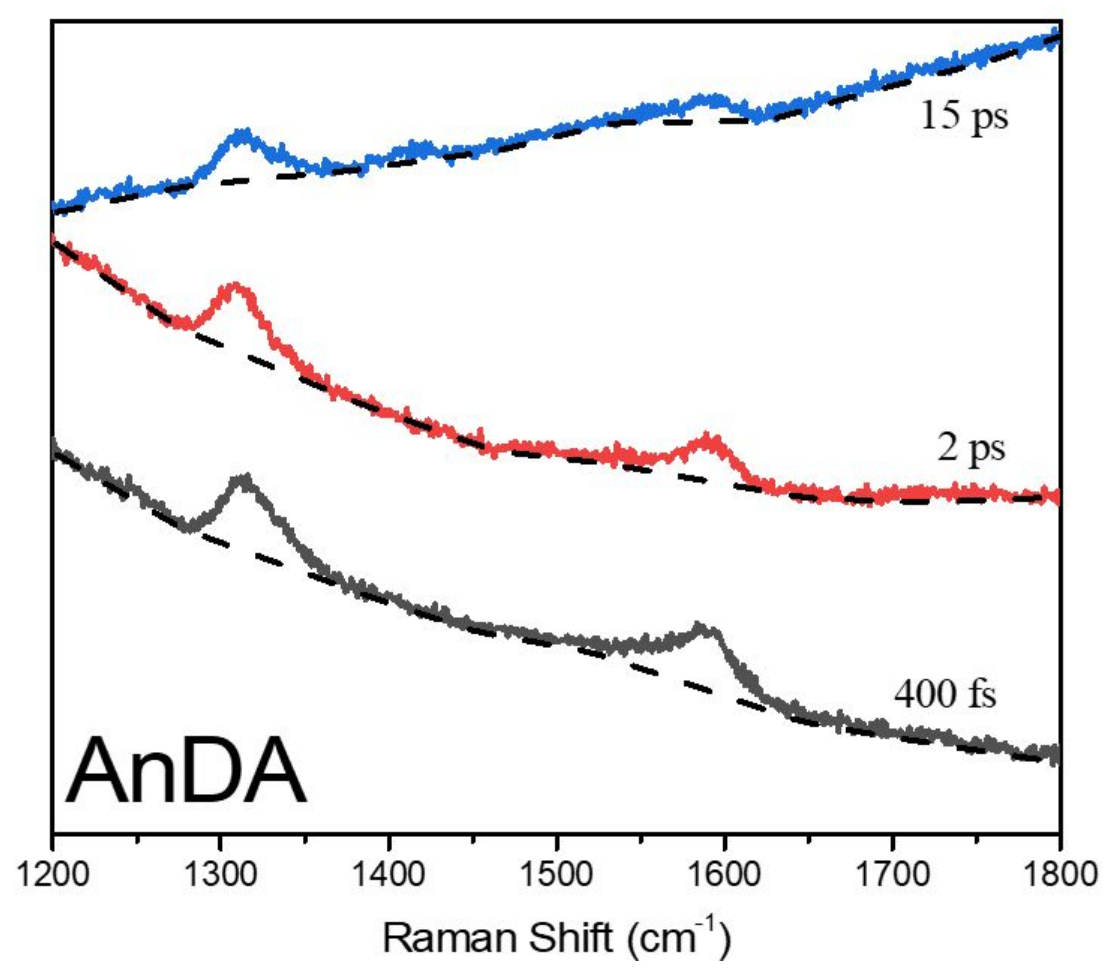

Figure S12. Excited state stimulated Raman data of AnDA at different time delays with the baseline drawn (black dot).

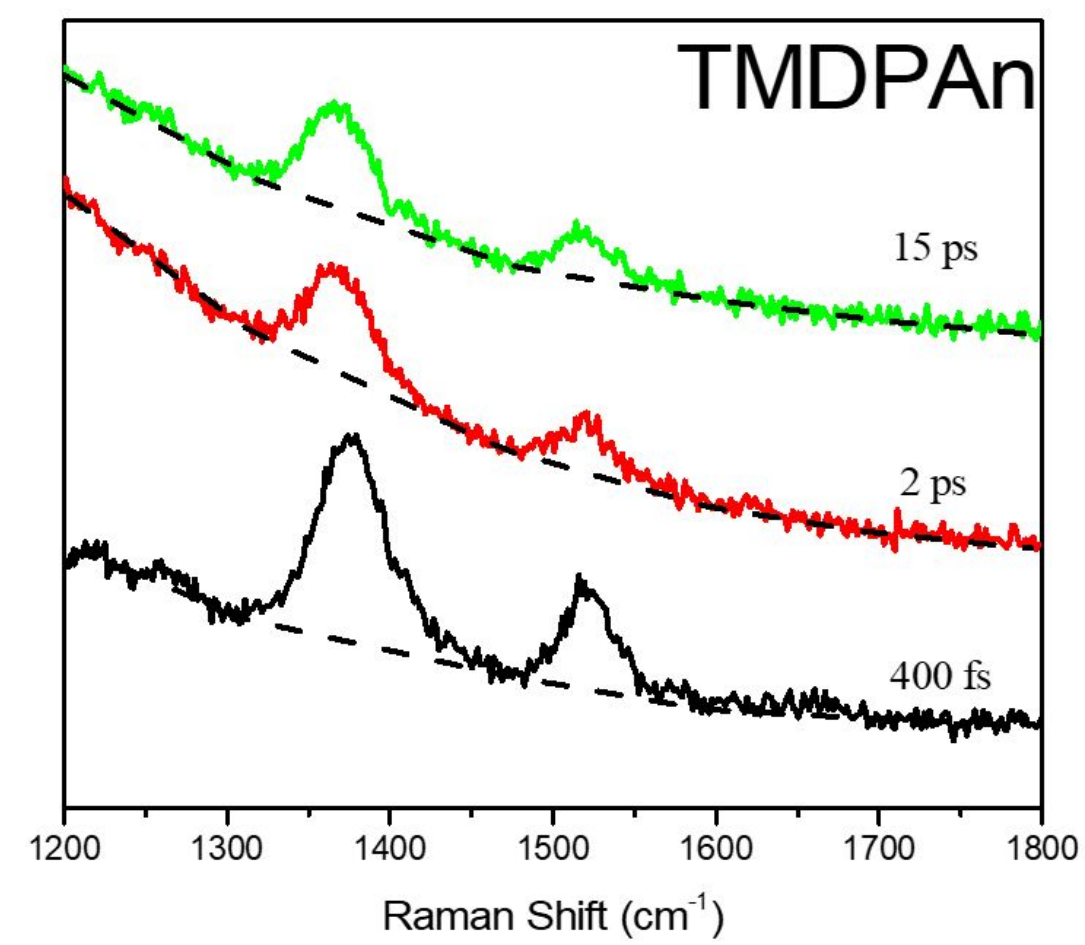

Figure S13. Excited state stimulated Raman data of TMDPAn at different time delays with the baseline drawn (black dot). 


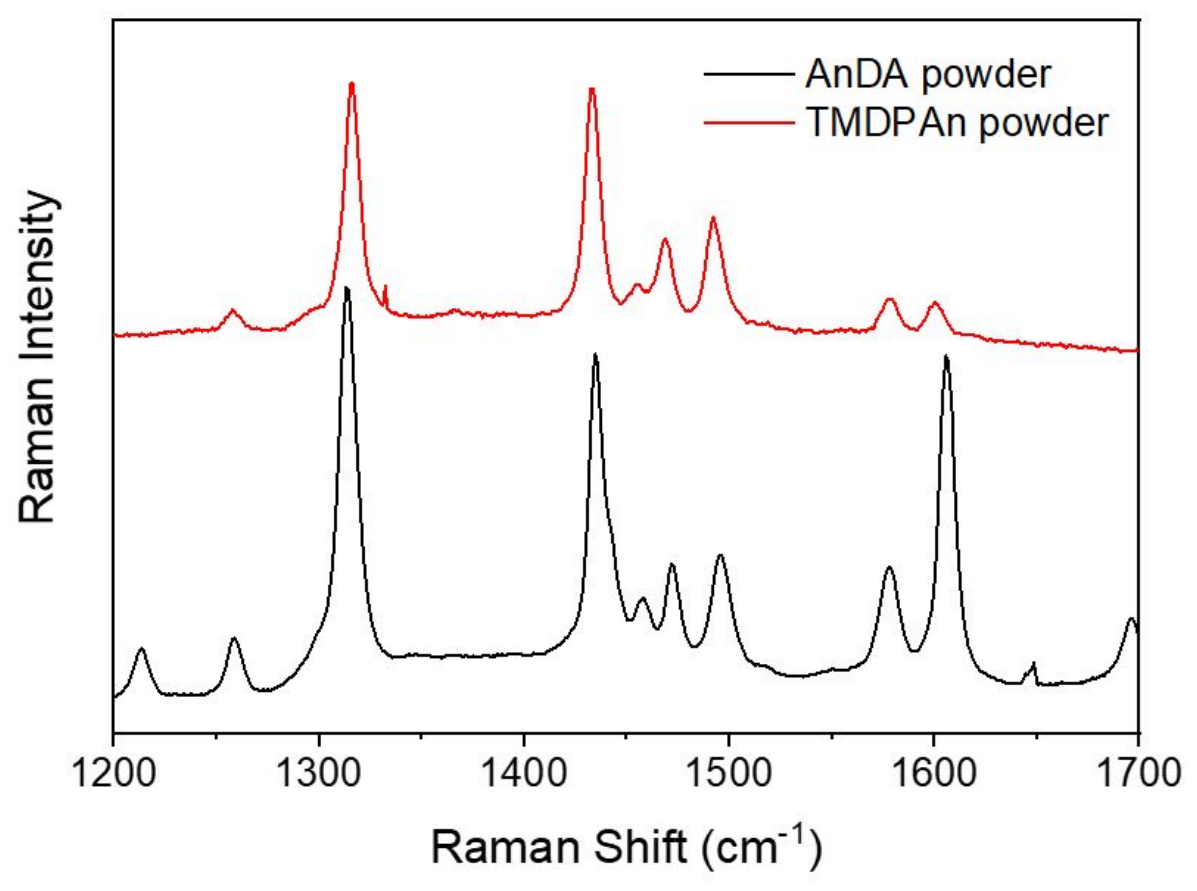

Figure S14. Ground state Raman spectra of powdered samples collected using commercial Raman Spectroscopy (Renishaw InVia). 

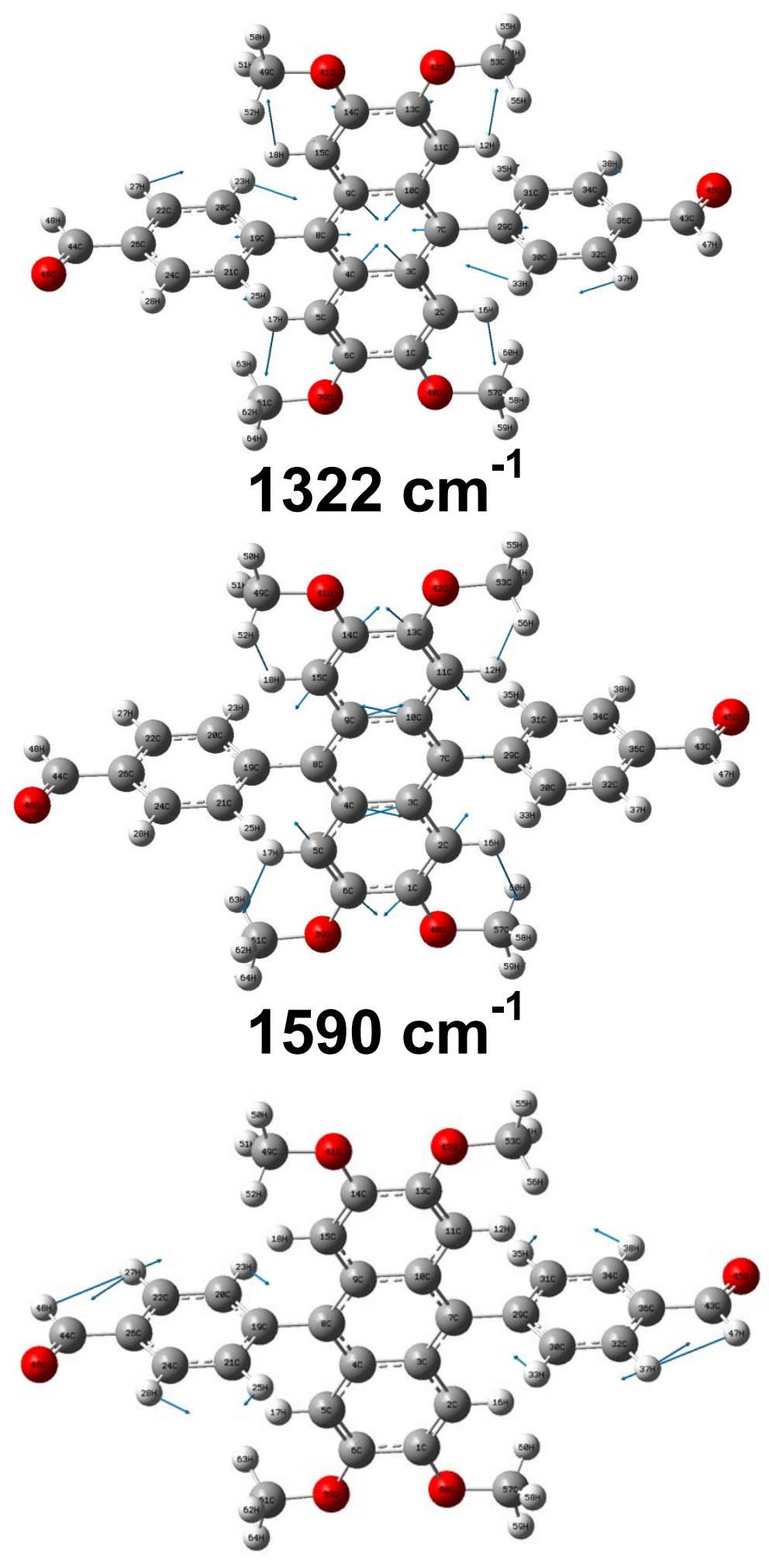

\section{$1417 \mathrm{~cm}^{-1}$}

Figure S15. DFT calculation (B3LYP) showing optimized structure and Raman frequencies of AnDA at $1322 \mathrm{~cm}^{-1}, 1590 \mathrm{~cm}^{-1}$ and $1417 \mathrm{~cm}^{-1}$. 


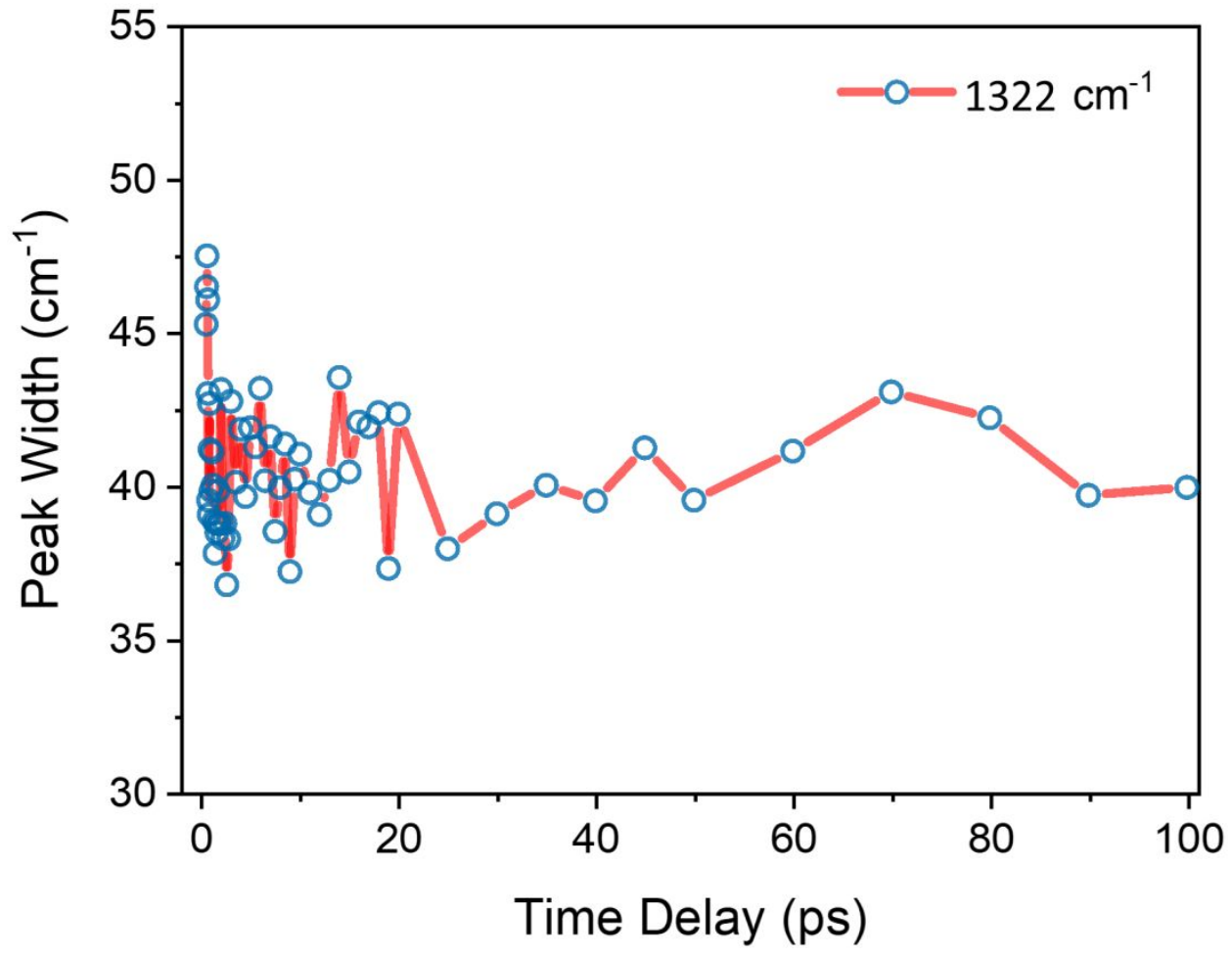

Figure S16. The linewidths obtained from the fit of the peak $1322 \mathrm{~cm}^{-1}$ as a function of delay time.

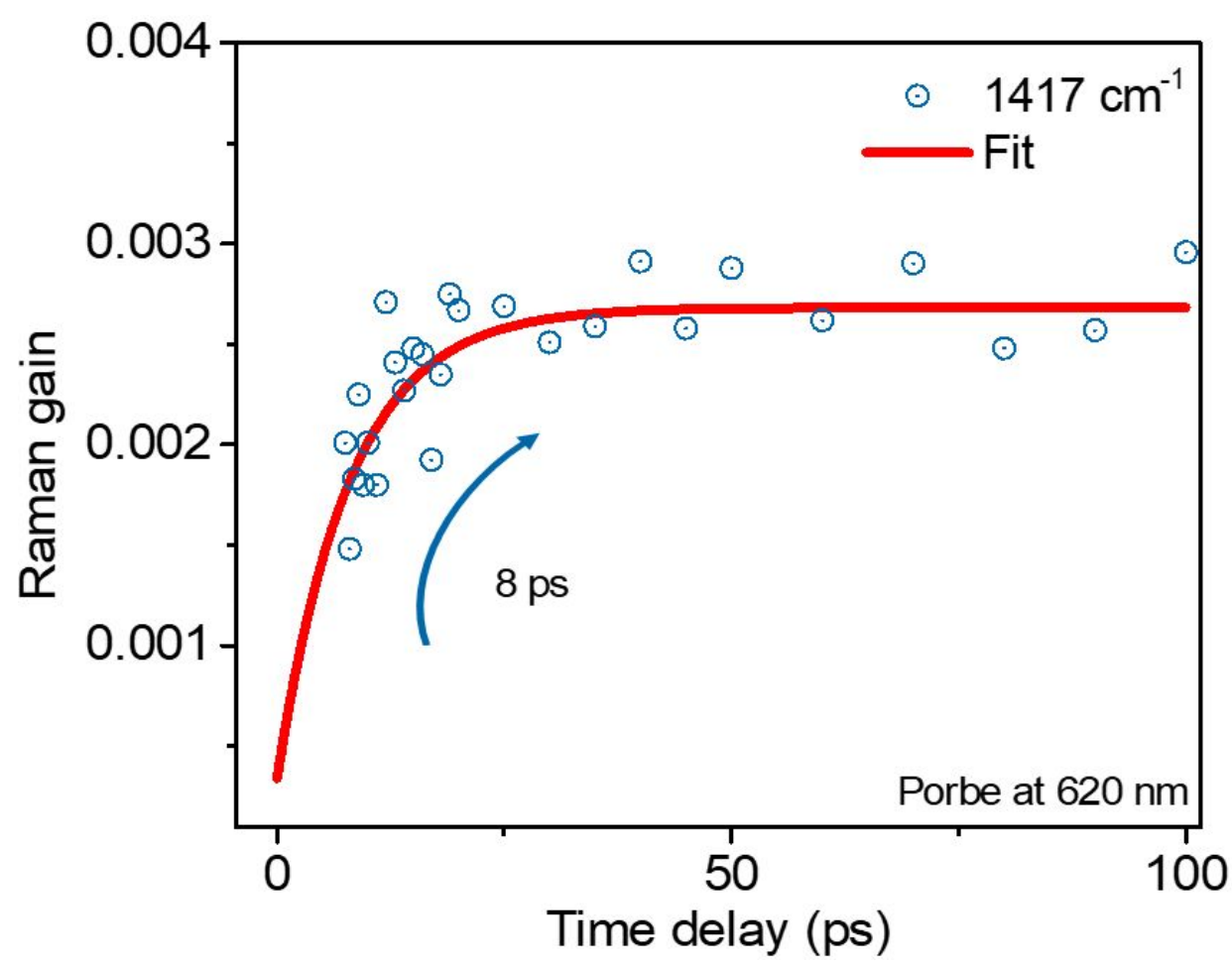

Figure S17. transient amplitudes of the vibrational modes of AnDA at $1417 \mathrm{~cm}^{-1}$. 

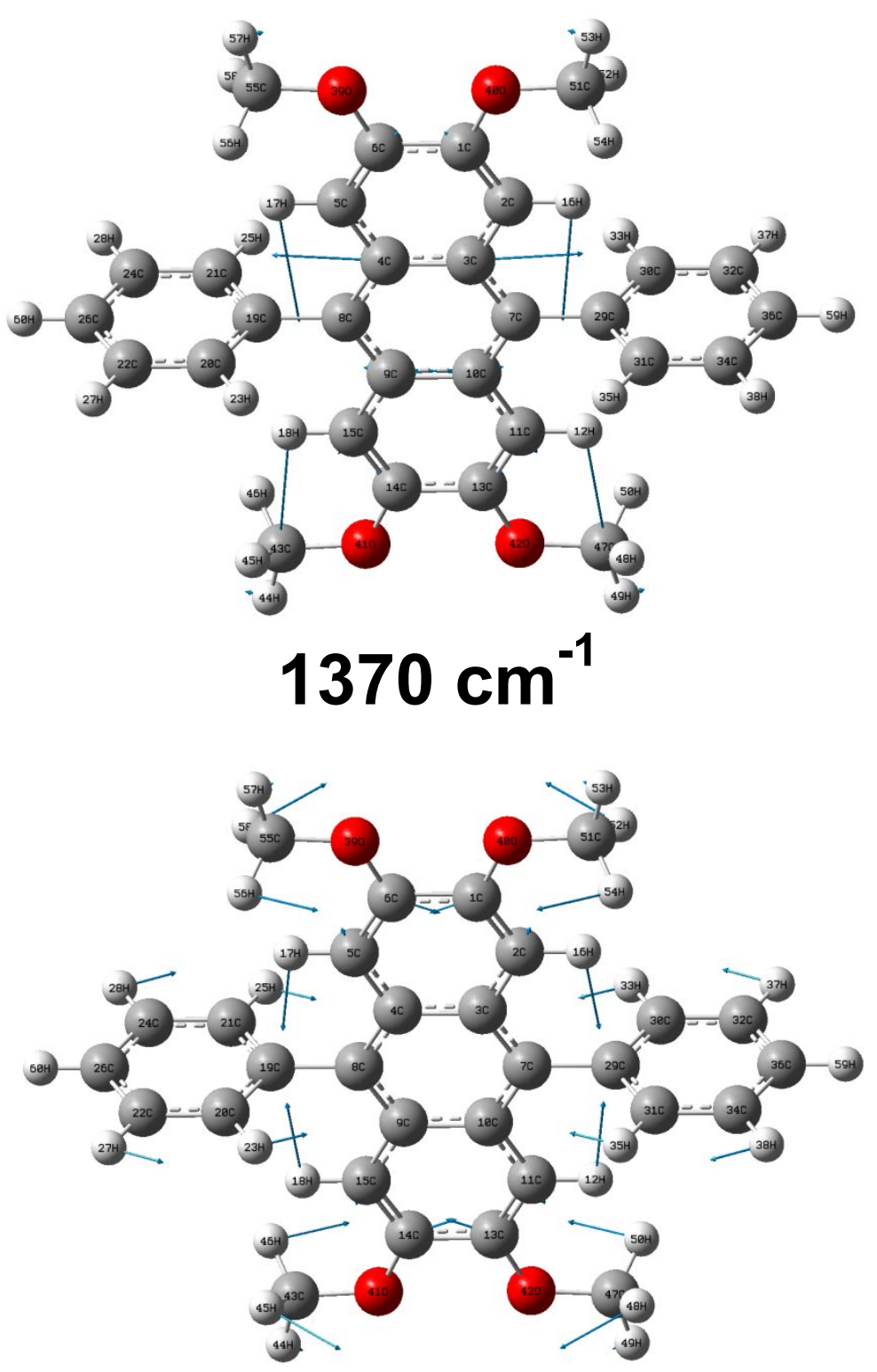

$1520 \mathrm{~cm}^{-1}$

Figure S18. DFT calculation (B3LYP) showing optimized structure and Raman frequencies of TMDPAn at $1370 \mathrm{~cm}^{-1}$ and $1520 \mathrm{~cm}^{-1}$. 


\section{Reference:}

(1) Frisch, M.; Trucks, G.; Schlegel, H. B.; Scuseria, G. E.; Robb, M. A.; Cheeseman,

J. R.; Scalmani, G.; Barone, V.; Mennucci, B.; Petersson, G. gaussian 09, Revision d. 01, Gaussian. Inc., Wallingford CT 2009, 201. 